\title{
Study of the MLB parameterisation for change in surface solar irradiance with sun zenith angle in clear sky
}

\author{
Z. Qu ${ }^{1}$, P. Blanc ${ }^{1}$, M. Lefèvre ${ }^{1}$, L. Wald ${ }^{1}$, and A. Oumbe ${ }^{2}$ \\ ${ }^{1}$ Centre for Energy and Processes, MINES ParisTech, BP207, 06904, Sophia Antipolis, France \\ ${ }^{2}$ German Remote Sensing Data Center, German Aerospace Center (DLR), \\ Postfach 1116, 82234 Wessling, Germany
}

Received: 21 December 2010 - Revised: 30 May 2011 - Accepted: 11 August 2011 - Published: 29 August 2011

\begin{abstract}
The MLB parameterisation (Modified Lambert-Beer, Mueller et al., 2004) describes the change in SSI with sun zenith angle (SZA) in clear-sky conditions. It applies to the direct and global SSI as well as their spectral distribution. We assess its performances by comparing its results to the outputs of the radiative transfer model libRadtran and standard interpolation procedures. The standard two-point fitting MLB function performs very well at SZA between $0^{\circ}$ and $60^{\circ}$ and fairly bad from $60^{\circ}$ to $89.9^{\circ}$. A parameterisation made of four MLBs for four intervals $\left(0^{\circ}, 60^{\circ}\right),\left(60^{\circ}, 75^{\circ}\right),\left(75^{\circ}, 85^{\circ}\right)$ and $\left(85^{\circ}, 89.9^{\circ}\right)$ is also tested. This piecewise MLB parameterisation exhibits satisfactory performances at any SZA and outperforms standard linear interpolation techniques. $95 \%$ of errors in global SSI are less than $1 \mathrm{~W} \mathrm{~m}^{-2}$ for each band and less than $5 \mathrm{~W} \mathrm{~m}^{-2}$ for total irradiance.
\end{abstract}

\section{Introduction}

A new direct method, Heliosat-4, is currently developed by the MINES ParisTech and the German Aerospace Center (DLR), aiming at estimating surface downwelling solar irradiance (SSI). The clear-sky module, an important part of this method, is based on the radiative transfer model (RTM) libRadtran (Mayer et al., 2010) and benefits from advanced products derived from recent Earth Observation missions (Oumbe et al., 2009). However, running a RTM in an operational mode is computer resources- and time-consuming. Mueller et al. (2004, 2009) suggest the use of parameterisations, among which the Modified Lambert-Beer function (MLB) to reduce the number of runs of RTM for the different sun zenith angles (SZA). Instead of calculating SSI values for each SZA with RTM runs, we use the MLB to interpolate SSI at any SZA by using SSI calculated by RTM at only two SZAs.

It is crucial that the interpolated SSIs obtained by the MLB are very close to those output from the RTM. The MLB applies to the direct and global components of SSI as well as their spectral distribution. The use of the MLB function is increasing in scientific papers. However, to our knowledge,

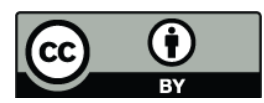

Correspondence to: $\mathrm{Z} . \mathrm{Qu}$

(zhipeng.qu@mines-paristech.fr) the performances of the MLB have not been fully reported, though a few results are presented in Mueller et al. (2004).

The novelty of this article is twofold: firstly to establish the performances of the MLB function by comparing its outcomes to those from libRadtran, and secondly, to propose an extended-MLB parameterisation to overcome the errors observed for large SZA. In addition, we have compared these performances to standard interpolation procedures in order to demonstrate the benefit of the MLB. It is assumed that libRadtran is delivering the actual values that should be reproduced.

\section{The Modified Lambert-Beer parameterisation}

Let $E_{\mathrm{TOA}, \lambda}\left(\theta_{s}\right)$ be the irradiance received by a horizontal plane at the top of atmosphere for the wavelength $\lambda$ and the SZA $\theta_{s}$. At altitude $z_{n}$, the direct spectral irradiance $E_{\lambda}\left(z_{n}, \theta_{s}\right)$ is a function of the optical depth $\tau_{\lambda}\left(z_{n}\right)$ and is given by the Lambert-Beer function (Liou, 1980):

$E_{\lambda}\left(z_{n}, \theta_{s}\right)=E_{\mathrm{TOA}, \lambda}\left(\theta_{s}\right) \cdot \exp \left(\frac{-\tau_{\lambda}\left(z_{n}\right)}{\cos \left(\theta_{\mathrm{s}}\right)}\right)$

The Modified Lambert-Beer function is based on Eq. (1) by adding a correction parameter $\alpha_{\lambda}\left(z_{n}\right)$ :

$E_{\lambda}^{\mathrm{MLB}}\left(z_{n}, \theta_{s}\right)=E_{\mathrm{TOA}, \lambda}\left(\theta_{s}\right) \cdot \mathrm{MLB}_{\lambda}\left(z_{n}, \theta_{\mathrm{s}}\right)$ 
$\operatorname{MLB}_{\lambda}\left(z_{n}, \theta_{\mathrm{s}}\right)=\exp \left(\frac{-\tau_{\lambda}\left(z_{n}\right)}{\cos \left(\theta_{\mathrm{s}}\right)^{\alpha_{\lambda}\left(z_{n}\right)}}\right)$

The two unknown parameters, $\alpha_{\lambda}\left(z_{n}\right)$ and $\tau_{\lambda}\left(z_{n}\right)$, are evaluated from two known irradiances $E_{\lambda}\left(z_{n}, \theta_{1}\right)$ and $E_{\lambda}\left(z_{n}, \theta_{2}\right)$. The fitting angles $\theta_{1}$ and $\theta_{2}$ are set here to $0^{\circ}$ and $60^{\circ}$ as in Mueller et al. (2004). Solving the linear system yields:

$\alpha_{\lambda}\left(z_{n}\right)=\frac{\ln \left(-\ln \left(\frac{E_{\lambda}\left(z_{n}, \theta_{1}\right)}{E_{\mathrm{TOA}, \lambda}\left(\theta_{1}\right)}\right)\right)-\ln \left(-\ln \left(\frac{E_{\lambda}\left(z_{n}, \theta_{2}\right)}{E_{\mathrm{TOA}, \lambda}\left(\theta_{2}\right)}\right)\right)}{\ln \left(\cos \left(\theta_{2}\right)\right)-\ln \left(\cos \left(\theta_{1}\right)\right)}$

$\tau_{\lambda}\left(z_{n}\right)=\exp \left(\ln \left(-\ln \left(\frac{E_{\lambda}\left(z_{n}, \theta_{1}\right)}{E_{\mathrm{TOA}, \lambda}\left(\theta_{1}\right)}\right)\right)+\alpha_{\lambda}\left(z_{n}\right) \cdot \ln \left(\cos \left(\theta_{1}\right)\right)\right)$

The fitting parameters $\alpha_{\lambda}\left(z_{n}\right)$ and $\tau_{\lambda}\left(z_{n}\right)$ are calculated respectively for direct and global SSI, and for each spectral band. Then, the diffuse SSI is calculated by subtracting the direct SSI to the global SSI.

\section{Assessing performances of the MLB}

The principle of the assessment is the following. A large set of SSI values is created by runs of the libRadtran: these are the reference SSI. We use the MLB function to compute the SSI for the same conditions and we compare them to the reference. The differences are synthesized by the means of standard quantities, such as the bias, the root mean square error (RMSE) or the percentile P95 of the frequency distribution. This is done for the direct, diffuse and global SSI for each of the 32 spectral bands of Kato et al. (1999) and the total SSI.

However, it should be noted that when dealing with the measurements, the deviations between MLB and ground measurements could be higher than those between MLB and libRadtran.

We use a Monte-Carlo technique to randomly select 1000 sets within the 8D-space defined by the 8 most influent inputs to libRadtran: aerosol optical thickness, type, and Angstrom coefficient, total column water, total column ozone, altitude of the ground, ground albedo and atmospheric profile. For each 8-tuple, libRadtran is run for 5 above surface altitudes - from 0 to $2 \mathrm{~km}$ by step $0.5 \mathrm{~km},-22 \mathrm{SZAs}$ - from 0 to $75^{\circ}$ by step $5^{\circ}$, from $75^{\circ}$ to $87.5^{\circ}$ by step $2.5^{\circ}$ and $89.9^{\circ}$. For a given 8 -tuple and a given above surface altitude, the SSI values at the fitting angles are used to compute the fitting parameters of the MLB. Then, the MLB function is used to compute the SSI for the other SZAs, and these assessed SSIs are compared to the reference SSIs.

We have weighted the random selection of the 8-tuple parameters by considering the average distribution in optical properties of the atmosphere. The probability density functions for all parameters, except aerosol optical thickness and Angstrom coefficient, and total column ozone, obey the uniform law. We have selected the chi-square law for aerosol

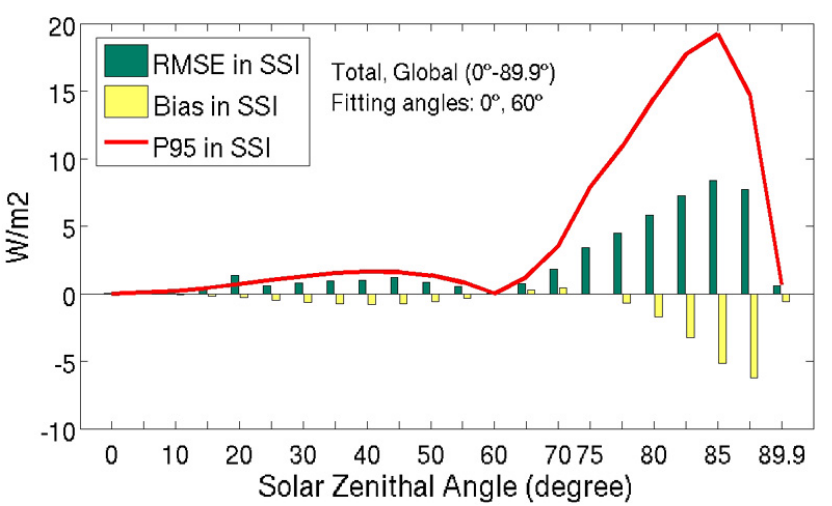

Figure 1. Errors in global total SSI $\left(0^{\circ}, 60^{\circ}\right)$.

optical thickness, the normal law for the Angstrom coefficient, and the beta law for total column ozone. The parameters of the laws have been empirically determined from the analyses of the observations made in the AERONET network for aerosol properties and from ozone products of the spatial missions OMI, GOME and SCHIAMACHY.

The results are illustrated in SSI or in clearness index $K T$ which is defined as follow:

$K T_{\lambda}\left(z_{n}, \theta_{s}\right)=E_{\lambda}\left(z_{n}, \theta_{s}\right) / E_{\mathrm{TOA}, \lambda}\left(\theta_{s}\right)$

Using $K T$ is a mean to show how good the method reproduces the effect of atmospheric contents on solar downwelling spectral irradiance, since the errors in SSI are expressed relatively to the irradiance at the top of atmosphere. Using $K T$ instead of SSI is more convenient in several cases and both should be used. For example, the magnitude of the SSI changes from one Kato band to another and it is difficult to compare the deviation in SSI for different Kato bands. On the other side, errors in $K T$ are commensurate and one can analyse and compare the errors for all cases more efficiently.

\section{Results}

\subsection{MLB with fitting angles: $0^{\circ}$ and $60^{\circ}$}

Figure 1 displays the errors in SSI. It depicts the error in the total global SSI as a function of the SZA. For each SZA, the bias, RMSE, and P95 are computed using all differences in SSI in a 2D-space (1000 atmospheric conditions and 5 above surface altitudes). The errors are small for SZA less than $60^{\circ}$ in full agreement with Mueller et al. (2004). $95 \%$ of errors (P95) are less than $2 \mathrm{~W} \mathrm{~m}^{-2}$ below $60^{\circ}$, whereas the P95 may reach $20 \mathrm{~W} \mathrm{~m}^{-2}$ at $85^{\circ}$. As for errors in spectral SSI for the Kato bands (not shown), they follow the same trend: for any band, the P95 is less than $1 \mathrm{~W} \mathrm{~m}^{-2}$ below $60^{\circ}$ and can be greater than $3 \mathrm{~W} \mathrm{~m}^{-2}$ between $75^{\circ}$ and $85^{\circ}$.

Figure 2 displays the error in the total direct SSI as a function of the SZA. Globally, the performance of MLB for the direct SSI largely surpasses that for the global SSI. 


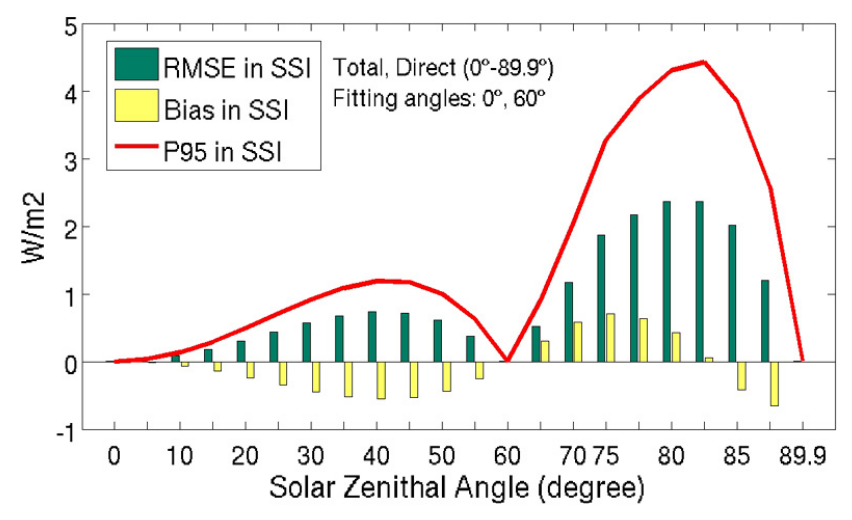

Figure 2. Errors in direct total SSI $\left(0^{\circ}, 60^{\circ}\right)$.

P95 is less than $1.5 \mathrm{~W} \mathrm{~m}^{-2}$ below $60^{\circ}$, whereas the $\mathrm{P} 95$ may reach $4 \mathrm{~W} \mathrm{~m}^{-2}$ at $82.5^{\circ}$. Similar results are attained for spectral bands (not shown): for any band, the P95 is less than $0.1 \mathrm{~W} \mathrm{~m}^{-2}$ below $60^{\circ}$ and attains its maximal value around $0.7 \mathrm{~W} \mathrm{~m}^{-2}$ between $80^{\circ}$ and $85^{\circ}$.

According to Figs. 1 and 2, between $0^{\circ}$ and $60^{\circ}$, the MLB performs very well for direct and global SSI, and thus for diffuse SSI. For greater SZAs, the error is much greater, especially for global SSI. Accordingly, the error in diffuse SSI is large. The MLB function tends to underestimate the diffuse SSI at large SZA, and therefore the global SSI, as demonstrated by the negative bias in Fig. 1. Similar conclusions are reached for each spectral band.

As discussed later and by Mueller et al. (2004), the usage of the modified Lambert-Beer function is physically motivated for direct irradiance; unsurprisingly, it does not perform very well for the diffuse irradiance. In addition, it is a fitting function and performs well within the range of SZA used for fitting and badly outside. Finally, the error in diffuse SSI or $K T$ increases with ground albedo because the diffuse fraction increases with the ground albedo. As expected, there is no influence of the ground albedo on the direct component.

\subsection{Piecewise MLB}

In order to reduce errors for large SZA, we have studied performances of the MLB function with more-spaced fitting angles, such as $\left(0^{\circ}, 70^{\circ}\right),\left(0^{\circ}, 75^{\circ}\right)$, or $\left(0^{\circ}, 80^{\circ}\right)$. In all cases, the performances were very poor and inacceptable. We have also tested other couples of fitting angles: $\left(60^{\circ}, 75^{\circ}\right)$ and $\left(75^{\circ}, 85^{\circ}\right)$. Expectedly, we observed that the errors are always small for SZA comprised between these closely-spaced fitting angles, but increase rapidly outside these intervals.

We study now the parameterisation made of four MLBs whose parameters are assessed for four intervals $\left(0^{\circ}, 60^{\circ}\right)$, $\left(60^{\circ}, 75^{\circ}\right),\left(75^{\circ}, 85^{\circ}\right)$, and $\left(85^{\circ}, 89.9^{\circ}\right)$. Now, we need 5 values of SSI for any situation. SSI at any SZA located in one of the four intervals is calculated by the MLB for this interval. Numerical errors may occur when solving Eqs. (4)

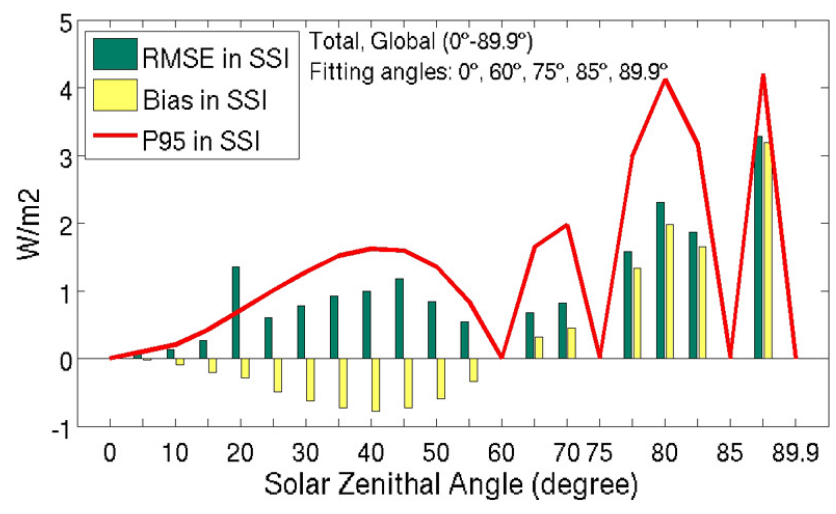

Figure 3. Errors for global total SSI (piecewise MLB).

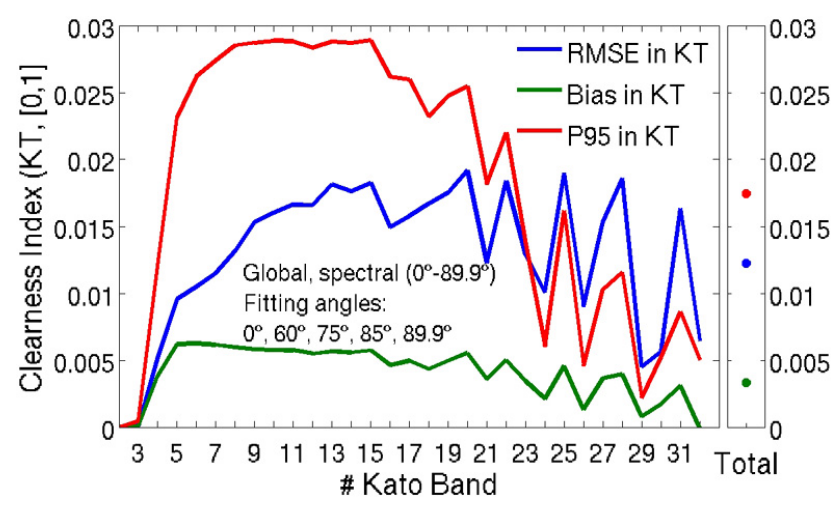

Figure 4. Errors for $K T$ glo for Kato bands and total (piecewise MLB).

and (5) at $89.9^{\circ}$. To ensure convergence, the clearness index for the direct SSI $K T$ dir is set to $1 \times 10^{-15}$ when it is less than this threshold.

Figure 3 exhibits the error in global total SSI as a function of the SZA. Global SSI shows greater errors than direct SSI (not shown). The bias, RMSE, and P95 errors are small. The maximum of RMSE, bias and P95 for all SZA is respectively less than $4 \mathrm{~W} \mathrm{~m}^{-2}, 4 \mathrm{~W} \mathrm{~m}^{-2}$ and $5 \mathrm{~W} \mathrm{~m}^{-2}$. We obtain most of the maximal errors in SSI when SZA equals to 87.5 .

Figure 4 displays the error in the clearness index of the global SSI $K T$ glo for the interval $\left[0^{\circ}, 89.9^{\circ}\right]$ for the Kato bands $3(295 \mathrm{~nm})$ to $32(4298 \mathrm{~nm})$ as well as the total. Errors are calculated in each band for all conditions. Errors are almost uniformly distributed for most of the Kato bands. The bias is very low, i.e., very low systematic error. The RMSE and P95 errors are small. The maxima of P95 are close to 0.030 , reached for bands $8-15(425-684 \mathrm{~nm})$; they are always less than $4 \%$ of the corresponding mean $K T$ glo. As for total, P95 is equal to 0.017 , i.e., less than $3 \%$ of the mean of $K T$ glo (0.68). Bias in SSI is negligible for each band and total, and P95 is less than $1 \mathrm{~W} \mathrm{~m}^{-2}$ for each band and $5 \mathrm{~W} \mathrm{~m}^{-2}$ for total. Errors in direct SSI are less than those for global SSI both in absolute and relative values. 


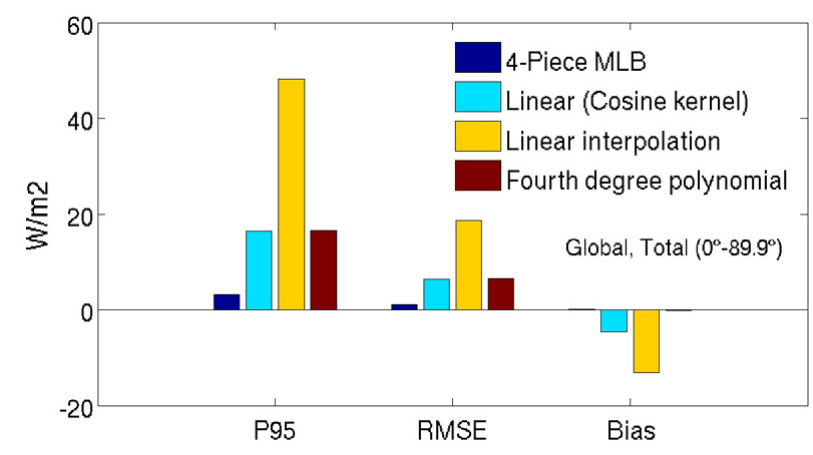

Figure 5. Comparison of errors for global total SSI (global performance of the four methods).

The clearness index is a convenient way to compare the results with different scales (large differences in SSI between Kato bands, and between Kato bands and total). In low SZA conditions, errors in SSI are generally small for Piecewise MLB (P95 $\left.<5 \mathrm{~W} \mathrm{~m}^{-2}\right)$ as what we showed in the Fig. 3. However, as SZA increases, the horizontal irradiance at the top of atmosphere decreases and a small error in SSI could lead to a large error in clearness index. These large errors in clearness index in high SZA conditions then disturb the global statistics. For example, the percentile 95 often locates in high SZA cases like $82.5^{\circ}$ where the error of 0.03 in clearness index is close to $5 \mathrm{~W} \mathrm{~m}^{-2}$ in global total SSI. Furthermore, the obtained RMSE in KT is less than 0.02 for each Kato band (Fig. 4), showing that deviations are generally much lower.

\section{Comparisons with other techniques}

We compared the performances of the piecewise MLB parameterisation with those attained by current interpolation techniques: linear interpolation, linear interpolation with cosine kernel between the same fitting angles: $0^{\circ}, 60^{\circ}, 75^{\circ}, 85^{\circ}$, $89.9^{\circ}$. In addition, considering that we need five SZA to define the piecewise MLB, we have tested the performances of a fourth degree polynomial. However, this method performs fairly badly for the large interval between $0^{\circ}$ and $60^{\circ}$. After multiple tests, we have selected five other fitting angles for the fourth degree polynomial: $0^{\circ}, 20^{\circ}, 45^{\circ}, 70^{\circ}$ and $89.9^{\circ}$. This set provides the best performances.

Figure 5 displays the performances of the four techniques for global total SSI for all conditions. This graph clearly demonstrates that the piecewise MLB outperforms the others. In terms of calculation time, the piecewise MLB is a bit slower than interpolation and has a significant advantage comparing to the fourth degree polynomial.

\section{Conclusions}

We have found that the MLB parameterisation (Mueller et al., 2004) is efficient for all wavelengths, provided the SZA ranges between $0^{\circ}$ and $60^{\circ}$. However, errors are large for SZA greater than $60^{\circ}$. The MLB function has a solid physical sense for the direct irradiance, and very good performances can be achieved. It is less physically sound for the diffuse irradiance and performances are lower for the diffuse and global irradiances.

After several tests, we have designed and validated a piecewise MLB parameterisation that offers very good performances for all wavelengths and all SZAs ranging from $0^{\circ}$ to $89.9^{\circ}$. Errors for direct SSI are less than those for global SSI in absolute and relative values. The bias for global SSI is less than $0.2 \mathrm{~W} \mathrm{~m}^{-2}$ for each band and less than $4 \mathrm{~W} \mathrm{~m}^{-2}$ for total irradiance. $95 \%$ of errors in global SSI are less than $1 \mathrm{~W} \mathrm{~m}^{-2}$ for each band and less than $5 \mathrm{~W} \mathrm{~m}^{-2}$ for total irradiance. The piecewise MLB parameterisation outperforms current linear interpolation, cosine-based techniques and forth degree polynomial.

Acknowledgements. The research leading to these results has received funding from the European Union's Seventh Framework Programme (FP7/2007-2013) under Grant Agreement no. 218793 (MACC project).

Edited by: E. Batchvarova

Reviewed by: three anonymous referees

SC nat $\boldsymbol{H}$ The publication of this article is sponsored by the Swiss Academy of Sciences.

\section{References}

Kato, S., Ackerman, T., Mather, J., and Clothiaux, E.: The kdistribution method and correlated-k approximation for shortwave radiative transfer model, J. Quant. Spectrosc. Ra., 62, 109121, 1999.

Liou, K. N.: An Introduction to Atmospheric Radiation, Academic Press, p. 182, 1980.

Mayer, B., Kylling, A., Emde, C., Buras, R., and Hamann, U.: libRadtran: library for radiative transfer calculations, Edition 1.0 for libRadtran version 1.5-beta, http://www.libradtran.org, 2 February 2010.

Mueller, R., Dagestad, K. F., Ineichen, P., Schroedter, M., Cros, S., Dumortier, D., Kuhlemann, R., Olseth, J. A., Piernavieja, G., Reise, C., Wald, L., and Heinnemann, D.: Rethinking satellite based solar irradiance modelling - The SOLIS clear sky module, Remote Sens. Environ., 91(2), 160-174, 2004.

Mueller, R. W., Matsoukas, C., Gratzki, A., Behr, H. D., and Hollmann, R.: The CM-SAF operational scheme for the satellite based retrieval of solar surface irradiance - A LUT based eigenvector hybrid approach, Remote Sens. Environ., 113, 10121024, 2009.

Oumbe, A., Blanc, Ph., Ranchin, T., Schroedter-Homscheidt, M., and Wald, L.: A new method for estimating solar energy resource, in: Proceedings of the ISRSE 33, Stresa, Italy, 4-9 May 2009, published by Joint Research Center, Ispra, Italy, USBKey, paper 773, 2009. 